\title{
State of Charge Estimation Algorithm for Unmanned Aerial Vehicle Power-Type Lithium Battery Packs Based on the Extended Kalman Filter
}

\author{
Haitao Zhang, Ming Zhou and Xudong Lan* \\ School of Aerospace Engineering, Tsinghua University, Beijing 100084, China; \\ zhanght15@mails.tsinghua.edu.cn (H.Z.); zmzlh@tsinghua.edu.cn (M.Z.) \\ * Correspondence: lanxd@mail.tsinghua.edu.cn; Tel.: +86-13810750789
}

\begin{abstract}
The lack of endurance is an important reason restricting further development of unmanned aerial vehicles (UAVs). Accurately estimating the state of charge (SOC) of the Li-Po battery can maximize the battery energy utilization and improve the endurance of UAVs. In this paper, the main current methods for estimating the SOC of vehicles were explored and discussed to unveil their advantages and disadvantages. In addition, the extended Kalman filter algorithm based on an equivalent circuit model was used to estimate SOC of power-type Li-Po batteries at different temperatures. The result showed that the closed-loop control method can effectively improve the battery life of small-sized electric UAVs.
\end{abstract}

Keywords: unmanned aerial vehicle; power-type Li-Po battery; state of charge; extended Kalman filter algorithm

\section{Introduction}

Small-sized unmanned aerial vehicles (UAVs) are widely used in various fields (including personal, commercial, and military fields) due to a series of advantages, i.e., small volume, light weight, flexibility, and low cost. A majority of small-sized UAVs apply an electric propulsion system and show a high practical application value due to having various characteristics, including simplicity in operation, high reliability, and ease of maintenance, storage, and transportation [1]. However, the energy density (about $185 \mathrm{~W} \cdot \mathrm{h} / \mathrm{kg}$ ) of a battery is far lower than that (about $10 \mathrm{~kW} \cdot \mathrm{h} / \mathrm{kg}$ ) of fuel oil. As a result, the battery life of electric UAVs is short [2]. Electric UAVs keep their mass unchanged during the entire mission period, which is different from oil engines, and the discharge voltage of the batteries constantly declines [3]. The discharge time of a battery is related to various factors, including discharge rate, power, and voltage [4]. Therefore, the accurate estimation of the state of the batteries is crucial for improving the utilization rate and increasing the battery life of electric UAVs.

Compared with lead-acid or nickel-metal batteries, lithium ( $\mathrm{Li}$ ) ion batteries show the following advantages, such as high specific energy, long cycle life, low self-discharge rate, no memory effect, and no pollution [5]. At present, Li-ion batteries are one of the most primary energy storage systems for small-sized UAVs with an electric propulsion system. However, Li-Po batteries still exhibit many shortcomings in practical applications. For example, Li-ion batteries with non-ideal security are prone to be affected by temperature (changing discharge rate), the service life of batteries is reduced due to deep discharges (lower than its nominal value) and according to the number of charge-discharge cycles, and then their performances are attenuated after aging. Single batteries show inconsistency after forming battery packs [6]. To solve these problems, it is necessary to favorably manage current battery packs. This is in addition to making improvements and development in these aspects, i.e., 
battery materials and manufacturing processes. Therefore, battery management systems (BMS) are emerging as a topic of research. BMS can effectively manage and control battery packs, ensure the safety and reliability of batteries during use, and prolong the practical service life of batteries while delivering their optimal performance [7].

Among the numerous functions of BMS, the estimation of the battery state is fundamental and crucial. The common battery states involve the state of charge (SOC), state of power (SOP), state of health $(\mathrm{SOH})$, and state of energy (SOE). SOC reflects the remaining capacity of batteries, which can generally be defined as the ratio of the remaining charge to the capacity (charge of a battery at full state of charge). SOC is an important internal state influencing the performance of batteries. The open-circuit voltage $(\mathrm{OCV})$, internal resistance, output power, remaining capacity, etc., are all closely related to SOC of the battery. Therefore, attaining accurate SOC is the basis for BMS to realize other related functions [8].

\section{Methods}

At present, there have been numerous researches on SOC estimation, which are mainly divided into six types.

(1) The ampere-hour (Ah) integration method is simple and easy to apply, so it is frequently used in BMS in the early stages. However, the method generally shows low estimation accuracy in practical application. The main reasons are summarized as follows: inaccurate initial value of the SOC will constantly influence the estimated result; the measurement error of the current sensors and the error of Coulombic efficiency lead to an accumulative error for the SOC; there is a large SOC estimation error when battery capacity parameters show errors. Thus, the SOC obtained by utilizing the Ah integration method alone exhibits a large error [9].

(2) The OCV method: owing to it requiring long-term standing, the simple OCV method is not applicable for dynamically estimating SOC online. Instead, it is usually used to assist and correct other methods (such as the Ah integration method). The OCV method based on models solves the problem. However, OCV estimation is greatly influenced by measurement noises of the measured current and voltage and model error. Therefore, there is low estimation accuracy in practical application $[10,11]$.

(3) Typical estimation methods based on the machine learning model include the neural network model [12] and the support vector regression model [13]. The machine learning model shows its superior universality, that is, it is unnecessary to consider types of batteries and the practical physical relationship between input and output values. However, the machine learning model depends on a large amount of data training, so it is only applicable within the original training data range. Where the practical application environment greatly differs from the training data, it fails to guarantee model accuracy and practical SOC estimation accuracy.

(4) Estimation methods based on an electrochemical model that concerns the electrochemical mechanism in batteries describes the dynamic characteristics of batteries from a microscopic scale. Compared with the equivalent circuit model that only describes external characteristics of batteries, the electrochemical model can offer more internal information on batteries and realize a high accuracy theoretically. Han [14] briefly introduced two commonly used electrochemical models-the single-particle (SP) model and pseudo-two-dimensional (P2D) model. As a battery model, the electrochemical model is more accurate theoretically compared with the equivalent circuit model. However, due to having more model parameters that make it difficult to conduct parameter matching, the electrochemical model is complex, less practically applied, and shows low accuracy.

(5) As a commonly used voltage model for batteries, the equivalent circuit model can describe the external characteristics of batteries by using basic components (including capacitors and resistors) to make up circuits based on the principle of batteries. Hu et al. [15] compared the accuracy, complexity, and robustness of 12 commonly used equivalent circuit models, which include the combined model, Rint model, first-order RC (resistor and capacitor) model, first-order RC model considering state delay, second- and third-order RC models. The research results showed that the first-order RC model is 
applicable for ternary Li-ion batteries (LiNixMnyCo1-x-yO $\mathrm{O}_{2}$ ), while the first-order $\mathrm{RC}$ model, which considers a state delay, can satisfy the requirements of a lithium iron phosphate $\left(\mathrm{LiFePO}_{4}\right)$ battery. Nejad et al. [16] compared ten battery models. By taking a ternary Li-ion battery and $\mathrm{LiFePO}_{4}$ battery as objects, the state estimation accuracies obtained by using different models were validated. The results revealed that the RC model exhibited better dynamic characteristics. For a majority of batteries, the second-order RC model is more applicable; the first-order RC model with a state delay is more suitable for $\mathrm{LiFePO}_{4}$ batteries. Overall, the equivalent circuit model, which is simple and accurate, is applicable to the practical environment.

(6) The extended Kalman filter algorithm (EKF) is a model-based closed-loop estimation method. As it regards the battery as a power system, the KF algorithm is used to estimate SOC as an internal state of the system. SOC estimation by using the KF algorithm can be divided into two links: priori estimation on the SOC of the battery by applying the Ah integration method; comparison of the terminal voltage predicted through the model with the measured terminal voltage. The difference between the two values is used for the posterior correction of the estimated result of the SOC. According to the process noise and observation noise of the system, the weight during posterior correction is automatically adjusted, that is, the level of Kalman gain. The common KF algorithm is suitable for linear systems. Cheng et al. [17] estimated the SOC of batteries by using the Rint model and the KF algorithm, and the estimation accuracy of their result significantly increases compared with that obtained through the Ah integration method based on OCV. However, the system of batteries is nonlinear. To apply a more accurate nonlinear battery model, it is necessary to use the EKF algorithm. Plett $[18,19]$ first proposed an estimation algorithm for the SOC based on the EKF. Afterward, SOC estimation was also conducted based on the EKF in many literatures. By taking ternary batteries and $\mathrm{LiFePO}_{4}$ batteries as research objects, Hu et al. [20] carried out SOC estimation by employing the EKF algorithm separately based on the first-order RC model and the first-order RC model with a state delay. They tested the robustness of the algorithm under different loads, temperatures, and degrees of attenuation. In the EKF algorithm, simple linearization mode is used; that is, first-order Taylor expansion is utilized to approximate nonlinear function. The sigma-point Kalman filter (SPKF) [21] and unscented Kalman filter (UKF) [22] improve the linearization process, and therefore, show better convergence and estimation accuracy in terms of optimal estimation of a highly nonlinear model. Additionally, to increase the applicability of algorithms, the corresponding adaptive Kalman filter (AKF) algorithm was also developed [23].

On the premise of having high model accuracy, this kind of closed-loop estimation method based on the equivalent circuit model, including the KF algorithm, exhibits high estimation accuracy for the SOC. For example, according to the results of previous research [24], SOC estimation accuracy can reach $2.5 \%$ when using a Luenberger observer. Zeng et al. [25] obtained the root-mean-square error within $0.5 \%$ for the estimated result of SOC by employing the AKF algorithm. Nevertheless, it is hard to guarantee the high estimation accuracy stated in the literature when practically applying the algorithms for the SOC. This is mainly because the performance and environment of batteries, the hardware of the BMS, etc., are not in an ideal state in practical applications; for example, various factors (such as battery aging, low-temperature environment, and measurement error of sensors) all likely lead to a reduction of actual SOC estimation accuracy. At present, only a part of the literature considers these influencing factors in SOC estimation. For example, some studies consider changes in model parameters and capacity after battery aging so as to synchronously estimate the SOC of the battery and capacity/model parameters by applying combined the KF or double KFs algorithm [26]. By utilizing recursive least squares, $\mathrm{Xia}$ et al. [27] realized the online identification of model parameters and applied the identification results to an SOC estimation.

Table 1 compares several methods that are commonly used at present. The above studies are mainly aimed at estimating the battery life of electric vehicles while research on SOC estimation of batteries for UAVs is hardly reported. In comparison, it can be seen that the closed-loop estimation method of the Kalman filter is relatively accurate and reliable, and the amount of calculation is moderate. It is the current mainstream method, but to improve the accuracy of actual SOC estimation in complex 
environments, it is also necessary to consider the influence of battery aging on model parameters such as capacity and internal resistance. In the study, the SOC estimation of Li-Po batteries of UAVs was conducted by utilizing an extended Kalman filter (EKF) algorithm-a closed-loop estimation method based on the equivalent circuit model.

Table 1. A comparison of advantages and disadvantages of the methods for estimating state of charge (SOC).

\begin{tabular}{lll}
\hline Estimation Method & Advantages & Disadvantages \\
\hline Ah integration & Simple and easy to be realized & $\begin{array}{l}\text { Greatly affected by initial SOC, } \\
\text { measurement error of current; } \\
\text { low accuracy }\end{array}$ \\
\hline OCV & Simple and accurate & Inapplicable for dynamic estimation \\
\hline $\begin{array}{l}\text { Typical estimation methods based } \\
\text { on a machine learning model }\end{array}$ & Universality & $\begin{array}{l}\text { Containing abundant internal applicable within the original } \\
\text { information of battery } \\
\text { training data range }\end{array}$ \\
\hline $\begin{array}{l}\text { Method based on the } \\
\text { Electrochemical model }\end{array}$ & $\begin{array}{l}\text { Simple and applicable for } \\
\text { dynamic estimation }\end{array}$ & $\begin{array}{l}\text { Complex model and huge } \\
\text { computational burden }\end{array}$ \\
\hline EKF & $\begin{array}{l}\text { Accurate and reliable; } \\
\text { ansensitive to measurement } \\
\text { noise and initial value of SOC }\end{array}$ & $\begin{array}{l}\text { Depends on moded by measurement } \\
\text { decreased estimation accuracy after } \\
\text { battery aging }\end{array}$ \\
\hline
\end{tabular}

\section{Modeling and Parameter Identification}

In this section, the power battery system was first modeled. Afterward, a hybrid pulse power characterization (HPPC) test was conducted, and parameters were identified based on the first-order RC model (equivalent circuit model). Finally, the principle of the EKF and its application in the established model were introduced.

\subsection{Modeling of Battery System}

According to Section 2, there are many studies on SOC estimation. The closed-loop estimation algorithm based on the equivalent circuit model with a moderate computational burden is applicable for practical application and shows advantages of accuracy and reliability, so it is a widely used method at present. According to numerous studies, in an ideal state, the closed-loop estimation algorithm based on the equivalent circuit model can reach a high estimation accuracy and can satisfy requirements of practical application. Shen [7] conducted parameter identification and verified the results based on the first-order and second-order RC models. It revealed that the estimation accuracy of the second-order $\mathrm{RC}$ model for voltage is slightly higher than that of the first-order RC model. However, the two models can both accurately simulate the voltage characteristics of batteries and show an insignificant difference in estimation accuracy. While taking the complexity of models into account, the first-order $\mathrm{RC}$ model lacks one RC parallel structure compared with the second-order RC model. Therefore, the first-order RC model is simpler and shows lower computation complexity. For consumptive flight control systems, the computing power is limited, and therefore, the first-order RC model (equivalent circuit model) was selected.

According to previous research results, for a ternary-graphite Li-ion battery, the circuit structure of the first-order RC model is shown in Figure 1.

The first-order $R C$ model consists of an ideal voltage source (represented by $O C V$ ), an Ohmic resistor $R_{0}$ and a parallel link of a resistor $R_{1}$ and a capacitor $C_{1}$. Where, $R_{1}$ and $C_{1}$ refer to the internal polarization resistor and polarization capacitor; the $R C$ link is used to describe the concentration polarization and electrochemical polarization characteristics of the battery. The current going through the Ohmic resistor is recorded as I, being positive during discharge. The voltage between the two 
ends of the internal polarization resistor and the output terminal voltage is recorded as $U_{1}$ and $U_{\text {out }}$, respectively; The time constant is $\tau_{1}=R_{1} C_{1}$. The formula for defining SOC and the equation for external characteristics of the first-order $R C$ model can be expressed as follows:

$$
\begin{gathered}
\operatorname{SOC}_{t}=\operatorname{SOC}_{t_{0}}-\frac{1}{C_{b a t}} \int_{t_{0}}^{t} \eta I d \tau, \\
U_{1}=I R_{1} \cdot\left[1-e^{-\frac{t}{\tau_{1}}}\right], \\
U_{\text {out }}=O C V-U_{1}-I R_{0},
\end{gathered}
$$

where $\eta$ denotes Coulombic efficiency, which generally approximates to $1 ; C_{b a t}$ stands for the capacity $(A \cdot h)$ of the battery under standard conditions; $S O C_{t}$ and $S O C_{t_{0}}$, respectively, represent the value at the time $t$ and $t_{0}$.

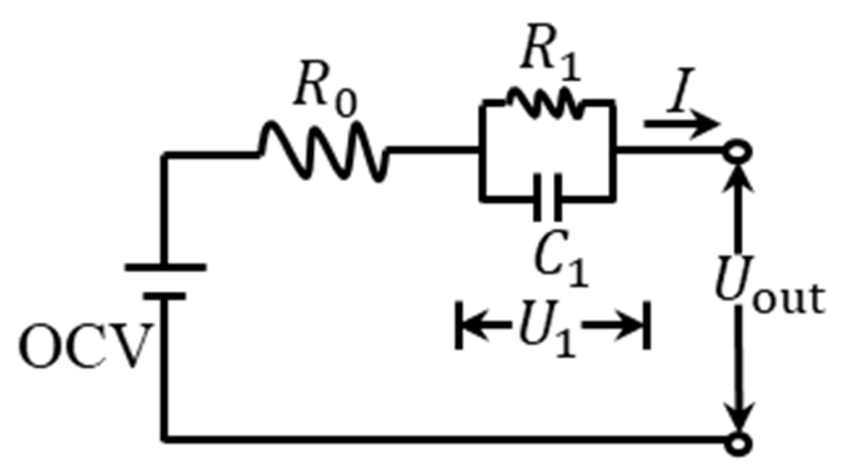

Figure 1. Circuit structure of the first-order RC (resistor and capacitor) model.

\subsection{HPPC Test and Parameter Identification}

By using a Li-ion battery as the research object, the fundamental performance parameters of the batteries used in the test are shown in Table 2.

Table 2. Performance parameters of Li-ion batteries used in the test.

\begin{tabular}{ll}
\hline Parameter & Value \\
\hline Nominal capacity & $1500 \mathrm{mAh}$ (standard charge/0.2C discharge, $2.75 \mathrm{~V}$ cut-off) \\
Nominal voltage & $3.7 \mathrm{~V}$ \\
Charge cut-off voltage & $4.2 \mathrm{~V}$ \\
Discharge cut-off voltage & $2.75 \mathrm{~V}$ \\
& $\mathrm{CC}-\mathrm{CV}(75 \mathrm{~mA}$ cut-off) \\
Standard charge method & Standard charge: $0.5 \mathrm{C}$ \\
& Rapid charge: $2 \mathrm{C}$ \\
& Charge: $0<\mathrm{T} \leq 10^{\circ} \mathrm{C}, 0.2 \mathrm{C}$ \\
Service temperature & $10<\mathrm{T} \leq 20^{\circ} \mathrm{C}, 0.5 \mathrm{C}$ \\
& $20<\mathrm{T} \leq 45^{\circ} \mathrm{C}, 1 \mathrm{C}$ \\
\hline
\end{tabular}

By utilizing an improved HPPC test method, a single battery was tested [28]. To be specific, a charge or discharge pulse was applied in the interval of $5 \%$ or $10 \%$ of the SOC (In particular, all SOCs in this experiment refer to relative SOC, that is, SOC = removable charge/actual battery capacity) in the process when the SOC of battery was changed from $100 \%$ to $0 \%$. As shown in Figure $2 \mathrm{a}$, after adjusting to a SOC point and keeping the battery standing for $1 \mathrm{~h}$, discharge pulse was successively applied for $30 \mathrm{~s}$; after standing for $40 \mathrm{~s}$, charge pulse was applied for $10 \mathrm{~s}$; afterward, the battery was discharged to the next SOC point, as shown in Figure $2 \mathrm{~b}$. To acquire characteristics of the battery at different temperatures, the HPPC test was separately carried out at $40^{\circ} \mathrm{C}, 25^{\circ} \mathrm{C}, 15^{\circ} \mathrm{C}$, and $5^{\circ} \mathrm{C}$. 


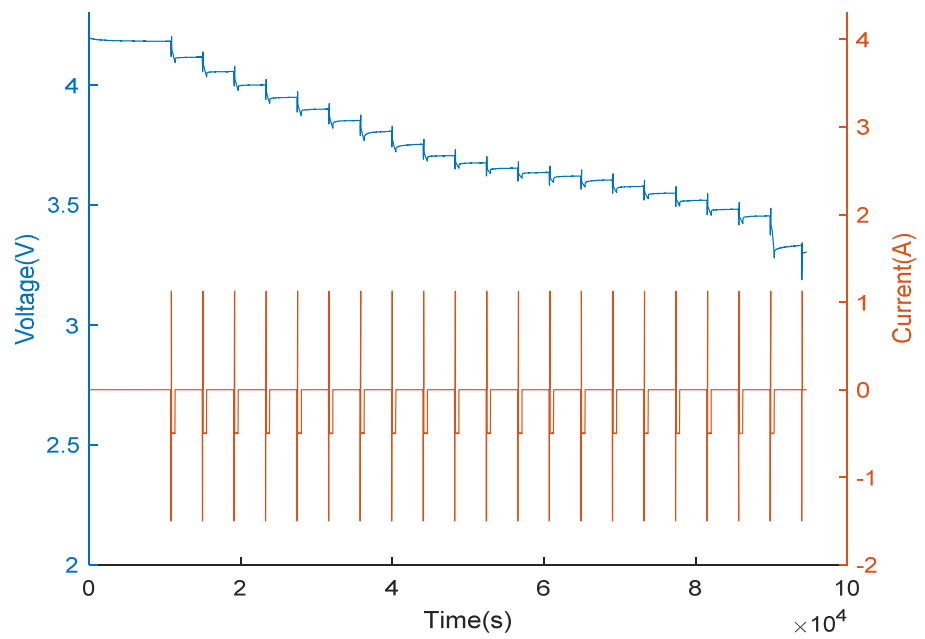

(a)

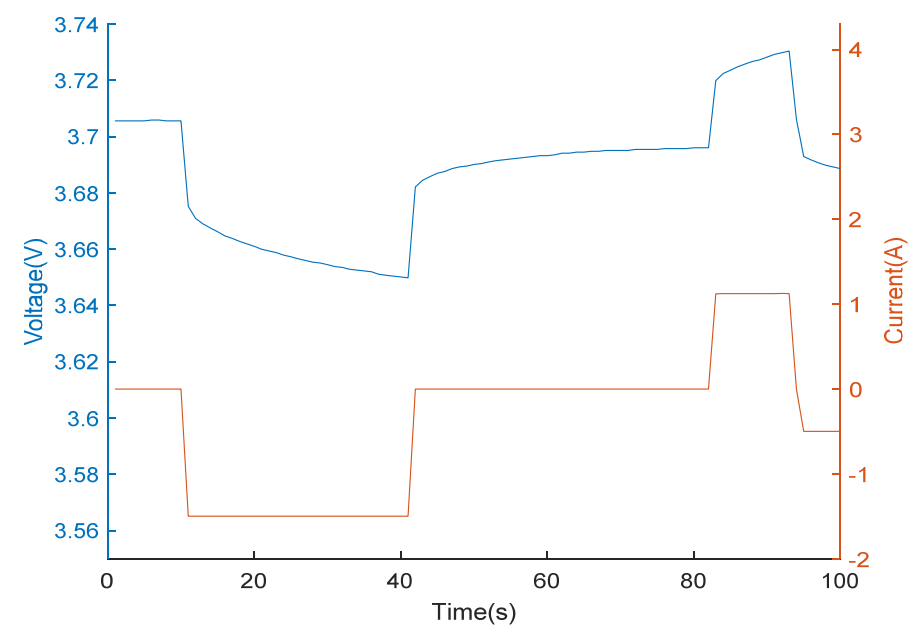

(b)

Figure 2. Hybrid pulse power characterization (HPPC) working conditions: (a) full working condition during HPPC in the range of $0 \%$ to $100 \%$ of the state of charge (SOC) at $25^{\circ} \mathrm{C}$; (b) local working condition during HPPC at $50 \%$ of the SOC at $25^{\circ} \mathrm{C}$.

In the test process of the battery, the charge and discharge were conducted on a Neware BTS-5V100A [29] test bench, and the test temperature was controlled by using an experimental temperature-controlled box produced by DROIDE Instrument (Beijing) Co., Ltd. For example, Figure 3:

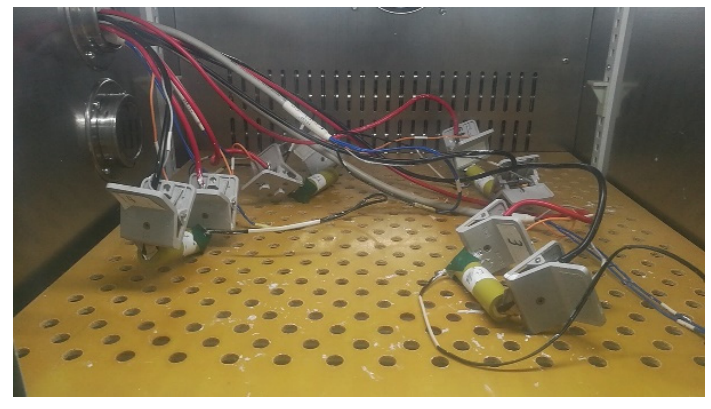

(a)

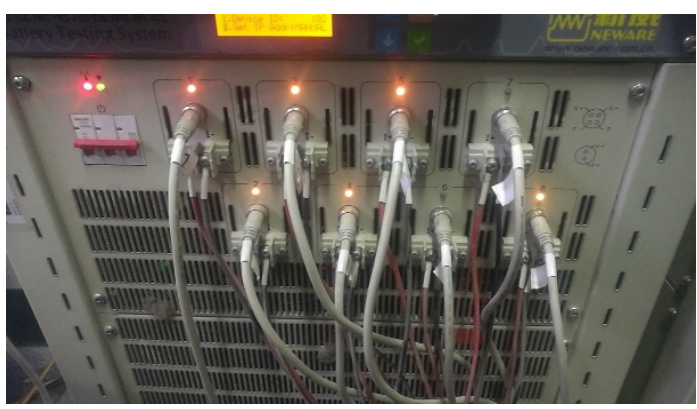

(b)

Figure 3. Off-line test bench: (a) Thermostat; (b) BTS-5V100A. 
The steps of the HPPC test are displayed in Figure 2. A charge or discharge pulse was applied to the battery at the interval of $5 \%$ or $10 \%$ of the SOC. Given a charge or discharge pulse can only trigger a small variation in the SOC, the SOC in each charge or discharge pulse can be considered as fixed. Therefore, by utilizing experimental data on voltage and current in a charge or discharge pulse, the model parameters at the corresponding SOC can be identified based on the optimal estimation method.

(1) During the HPPC test, the battery stood at each SOC point, and therefore, it was feasible to directly read the voltage after standing as the OCV of the battery. Figure 4 presents the OCV-SOC curves at different temperatures obtained based on test data. It can be seen that the OCV of the Li-ion batteries used in the test insignificantly varied with temperature. However, it can be found that with the reduction in temperature, the corresponding voltage of the same SOC point slightly declined. It was worth noting that the HPPC test was ended owing to the capacity of battery being reduced to $5 \%$ of the SOC at $15{ }^{\circ} \mathrm{C}$, while HPPC had ended at $10 \%$ of SOC at $5{ }^{\circ} \mathrm{C}$.

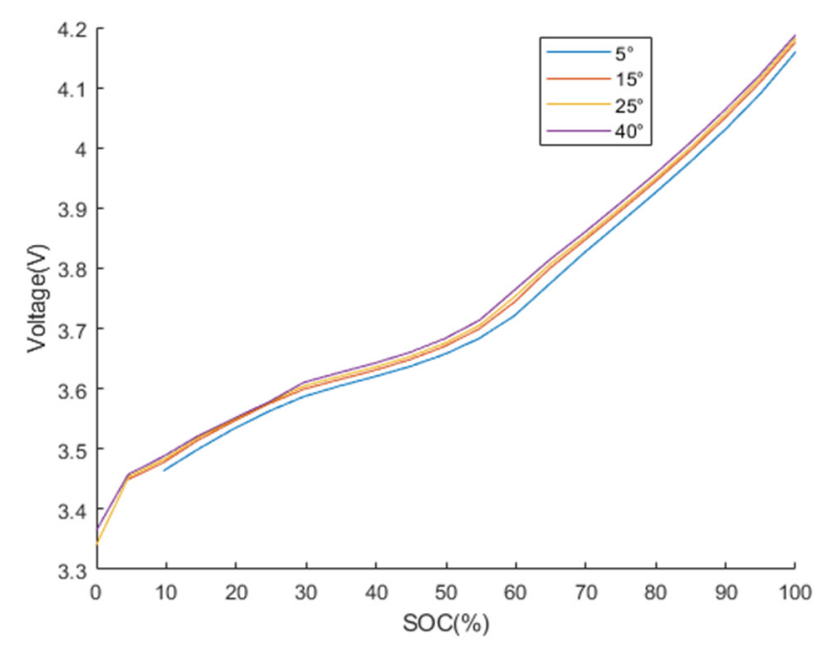

Figure 4. Open-circuit voltage-state of charge (OCV-SOC) curves.

(2) According to the change in voltages of batteries before and after applying charge and discharge pulses during the HPPC test, it was easy to calculate the direct-current (DC) internal resistance of the batteries during charge and discharge. Figure 5 shows the DC internal resistances of the batteries at different temperatures after discharge for $1 \mathrm{~s}$. As shown in Figure 5, the internal resistance of the batteries significantly changed with temperature: The internal resistance of the batteries obviously rose with decreasing temperature. For the condition with a large SOC $(20 \%<$ SOC $<100 \%)$, the internal resistance insignificantly changed with SOC; at low SOC $(<20 \%)$, the internal resistance of the batteries remarkably increased with reducing SOC.

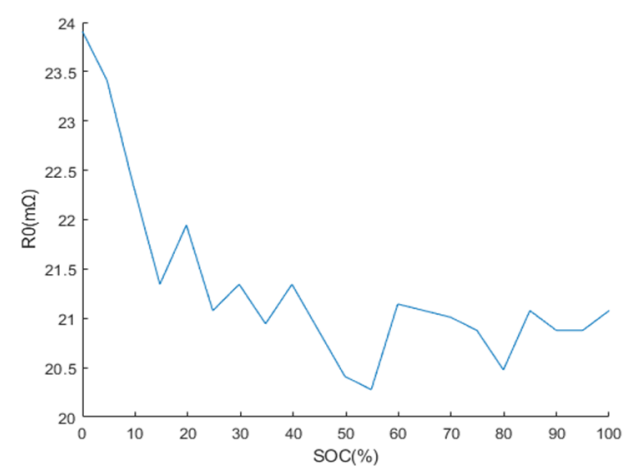

(a)

Figure 5. Cont. 


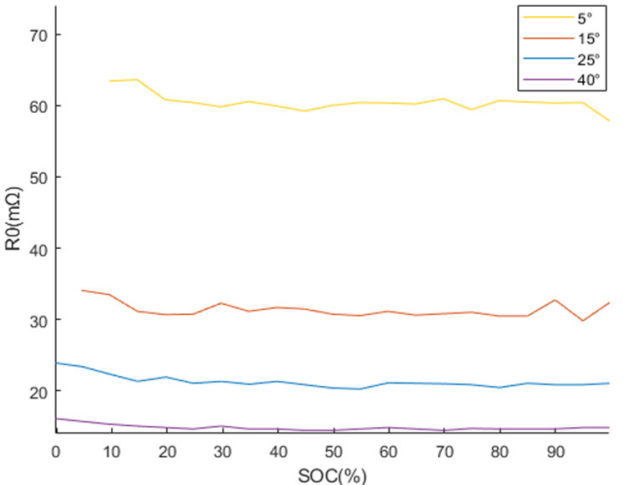

(b)

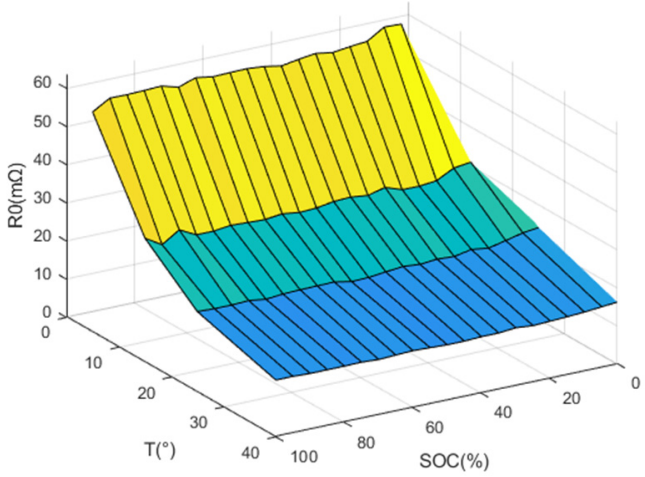

(c)

Figure 5. DC internal resistance $R_{0}$ during discharge: (a) DC internal resistance at $25^{\circ} \mathrm{C}$; (b) DC internal resistances at different temperatures; (c) MAP of DC internal resistance about temperature $\mathrm{T}$ and SOC.

(3) SOC estimation was carried out by using the first-order RC model. Based on HPPC test data at different temperatures, the model parameters at different temperatures can be identified by conducting 15-order polynomial fitting, thus finally attaining the 3D Map between model parameters SOC and temperature. Figures 6 and 7 show the identification results of polarization internal resistance and capacitance, respectively.

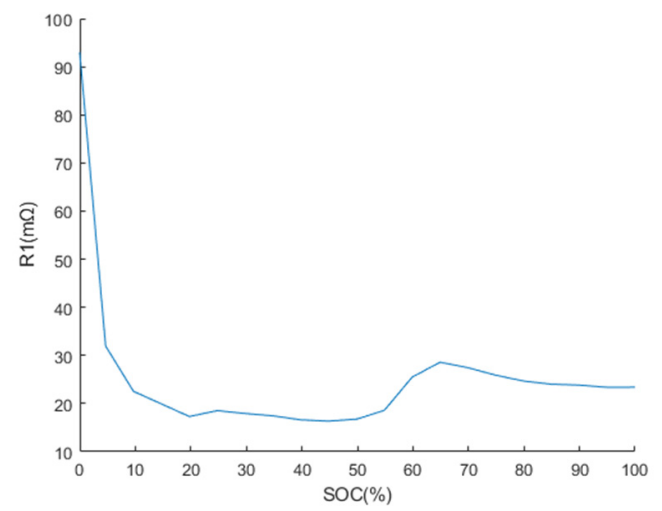

(a)

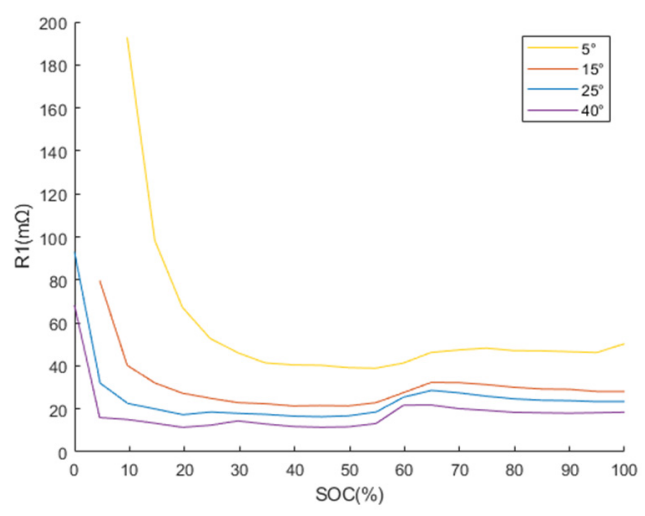

(b)

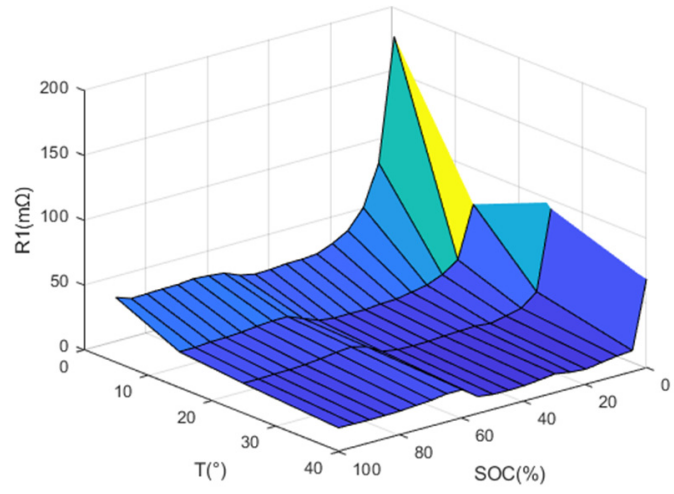

(c)

Figure 6. Polarization internal resistance $R_{1}$ : (a) polarization internal resistance at $25^{\circ} \mathrm{C}$; (b) polarization internal resistances at different temperatures; (c) MAP of polarization internal resistance about temperature $\mathrm{T}$ and SOC. 


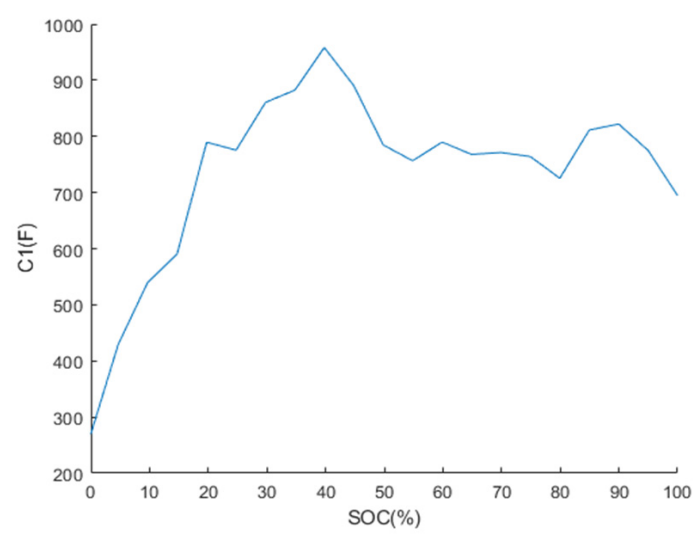

(a)

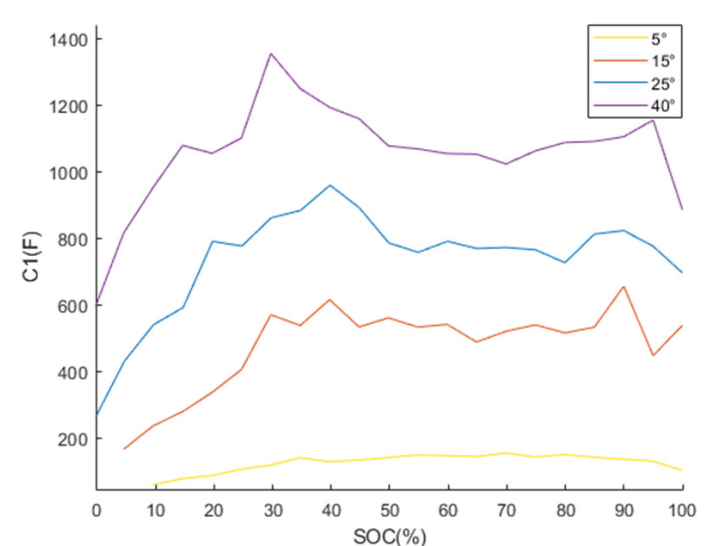

(b)

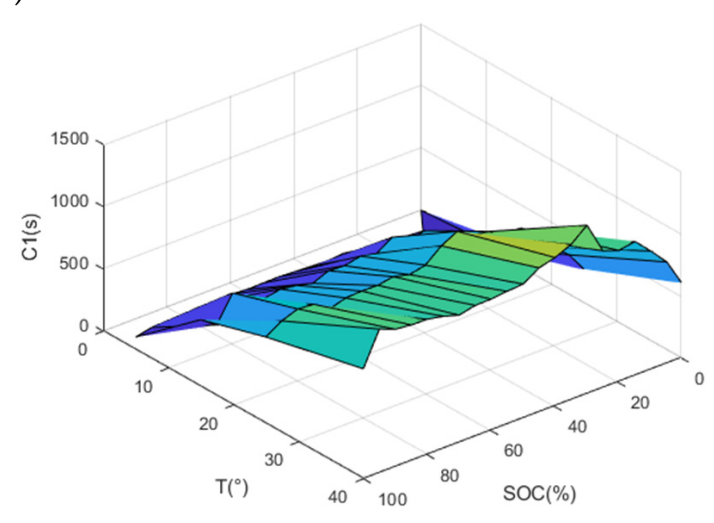

(c)

Figure 7. Polarization capacitance $C_{1}$ : (a) polarization capacitance at $25^{\circ} \mathrm{C}$; (b) polarization capacitances at different temperatures; (c) MAP of polarization capacitance about temperature T and SOC.

\subsection{EKF Algorithm}

A dynamic nonlinear system generally can be described by using the following state space model.

$$
\begin{gathered}
x_{k+1}=f\left(x_{k}, u_{k}\right)+w_{k}, \\
y_{k}=g\left(x_{k}, u_{k}\right)+v_{k},
\end{gathered}
$$

where, Formulae (4) and (5) separately refer to the state and output equations of the system; $x_{k}, u_{k}$ and $y_{k}$ denote the state vector in system at the time $k$, input and output vectors of system, respectively; $w_{k}$ represents random process noise or disturbance, which reflects some unmeasured inputs influencing the state of system, and $v_{k}$ stands for sensor noise, reflecting the measurement error of system output. The KF algorithm, as an optimal estimation algorithm, makes the optimal estimation on the internal state of the system in the sense of minimum variance by using the state equation according to observed input and output data of the system. The common KF algorithm is only applicable for a linear system; however, a battery belongs to a nonlinear system. Therefore, the SOC estimation was conducted by applying the EKF algorithm. Plett $[18,19]$ made a detailed introduction and analysis very early on the EKF algorithm and its application in the estimation of the battery state. The initialization process and iterative estimating equation of the KF algorithm are displayed in Table 3. 
Table 3. The iterative equation used for the EKF algorithm.

\begin{tabular}{ll}
\hline Definition: & \\
\hline$\hat{A}_{k}=\left.\frac{\partial f\left(x_{k}, u_{k}\right)}{\partial x_{k}}\right|_{x_{k}=\hat{x}_{k}^{+}}$ & $\hat{C}_{k}=\left.\frac{\partial g\left(x_{k}, u_{k}\right)}{\partial x_{k}}\right|_{x_{k}=\hat{x}_{k}^{-}}$ \\
\hline Initialization: & \\
Under k $=0$, it is supposed that, & $\Sigma_{\tilde{x}_{, 0}}^{+}=E\left[\left(x_{0}-\hat{x}_{0}^{+}\right)\left(x_{0}-\hat{x}_{0}^{+}\right)^{T}\right]$ \\
$\hat{x}_{0}^{+}=E\left[x_{0}\right]$ & \\
\hline Iterative Calculation: & \\
Under k $=1,2 \ldots$ & $\hat{x}_{k}^{-}=f\left(\hat{x}_{k-1}^{+}, u_{k-1}\right)$ \\
Update of state vector: & $\Sigma_{\widetilde{x}, k}^{-}=\hat{A}_{k-1} \Sigma_{\widetilde{x}, k-1}^{+} \hat{A}_{k-1}^{T}+\Sigma_{w}$ \\
Update of time of error covariance matrix: & $L_{k}=\Sigma_{\widetilde{x}, k}^{-} \hat{C}_{k}^{T}\left[\hat{C}_{k} \Sigma_{\widetilde{x}, k}^{-} \hat{C}_{k}^{T}+\Sigma_{v}\right]^{-1}$ \\
Calculating Kalman gain: & $\hat{x}_{k}^{+}=\hat{x}_{k}^{-}+L_{k}\left[y_{k}-g\left(\hat{x}_{k}^{-}, u_{k}\right)\right]$ \\
Updating measurement of state vector: & $\Sigma_{\widetilde{x}, k}^{+}=\left(I-L_{k} \hat{C}_{k}\right) \Sigma_{\tilde{x}, k}^{-}$ \\
\hline Updating measurement of error covariance matrix:
\end{tabular}

Where, $\hat{A}_{k}$ and $\hat{C}_{k}$ separately refer to the first-order Taylor expansion coefficients of state and output equations, called coefficient matrixes; $L_{k}$ denotes Kalman gain, and $\Sigma_{w}$ and $\Sigma_{v}$ separately stand for covariance matrixes of input and output measurement noises $w_{k}$ and $v_{k} ; \Sigma_{x, k}$ represents the covariance matrix of error of state estimation, showing the uncertainty of state estimation. For discrete KF algorithm, state vector and its covariance matrix are subjected to two updates in each iterative step. The first update of time is priori estimation based on state equation, in which the estimated results are expressed as $\hat{x}_{k}^{-}$and $\Sigma_{\widetilde{x}, k^{\prime}}^{-}$, respectively; the second update of measurement is posterior correction based on measured values and the corrected states are represented as $\hat{x}_{k}^{+}$and $\Sigma_{\tilde{x}, k^{\prime}}^{+}$respectively.

For SOC estimation of batteries, SOC is an internal state which needs to be estimated. Generally, it is thought that current is input and terminal voltage is output of the system. Therefore, based on Equation (1) to (3), the state Equation (6) about SOC and the output Equation (7) of the system are attained, where, $w_{k}$ and $v_{k}$ denote input and output noises, respectively.

$$
\begin{gathered}
{\left[\begin{array}{c}
\operatorname{SOC}(k+1) \\
u_{1}(k+1)
\end{array}\right]=\left[\begin{array}{cc}
1 & 0 \\
0 & e^{\frac{-T}{R_{1} C_{1}}}
\end{array}\right]\left[\begin{array}{c}
\operatorname{SOC}(k) \\
u_{1}(k)
\end{array}\right]+\left[\begin{array}{c}
\eta \frac{T}{C_{b a t}} \\
R_{1}\left(1-e^{\frac{R_{1} C_{1}}{R_{1}}}\right)
\end{array}\right] i(k)+w_{k}} \\
U_{\text {out }}(k)=U(\operatorname{SOC}(k))-u_{1}(k)-i(k) R_{0}+v_{k}
\end{gathered}
$$

where:

$w_{k}$ is the input noise matrix, caused by the error of the model;

$v_{k}$ is the measure noise matrix, caused by battery voltage measurement error;

$T$ is the sampling period;

$w_{k}$ and $v_{k}$ are irrelevant.

According to the state space model for general systems and the calculation formula for coefficient matrix in Table 3 , the state vector $x_{k}$, system output $y_{k}$ and input $u_{k}$ as well as corresponding coefficient matrixes $\hat{A}_{k}$ and $\hat{C}_{k}$ for SOC estimation by using EKF algorithm are shown as follows:

$$
\begin{gathered}
x_{k}=\left[\operatorname{SOC}_{k}, u_{1, k}\right]^{T}, \\
y_{k}=U_{o u t, k \prime}, \\
U_{k}=I_{k},
\end{gathered}
$$




$$
\begin{gathered}
\hat{A}_{k}=\left.\left[\begin{array}{cc}
1 & 0 \\
0 & \exp \left(-T / R_{1, k} C_{1, k}\right)
\end{array}\right]\right|_{S O C=S O \hat{O} C_{k}^{+}} \\
\hat{C}_{k}=\left[\left.\frac{d O C V}{d S O C}\right|_{S O C=S O C_{k}^{-\prime}}-1\right] .
\end{gathered}
$$

As a result, the models and corresponding equations for estimating the SOC of power-type Li batteries are established.

\section{Experimental Verification and Discussion}

According to the iterative formulae in Table 3, the state vectors, including SOC, are subjected to iterative estimation, and the estimation process is shown in Figure 8. It can be easily seen that SOC estimation using the EKF algorithm is essentially the preliminary estimation of the SOC value according to current integration, followed by a closed-loop correction of the estimated SOC taking voltage as feedback signals based on the battery model. The feedback coefficient is exactly the Kalman gain, which is automatically determined according to the optimal control theory by using the EKF algorithm.

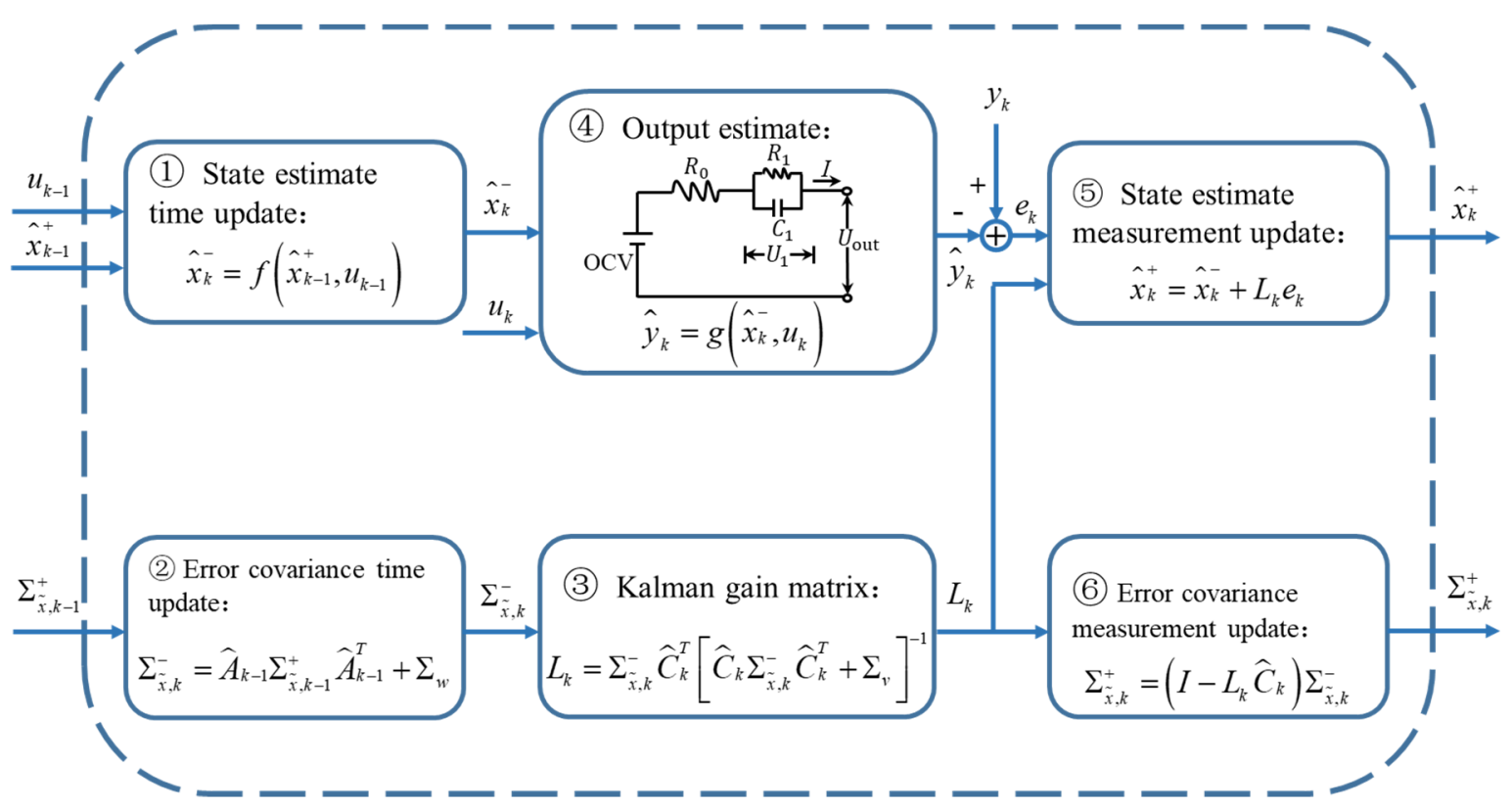

Figure 8. SOC estimation based on the extended Kalman filter (EKF) algorithm.

According to the aforementioned estimation algorithms for the SOC, the estimation model for SOC can be established in the MATLAB/Simulink platform. To verify the accuracy of estimation algorithms for the SOC, the tests on dynamic working conditions at four temperatures $\left(40^{\circ} \mathrm{C}, 25^{\circ} \mathrm{C}, 15^{\circ} \mathrm{C}\right.$, and $5{ }^{\circ} \mathrm{C}$ ) were carried out, and SOC estimation was validated based on test data [29].

The results of offline bench testing on simulated working conditions are presented in Figure 9.

SOC estimation was carried out based on measured data, and the obtained estimated results are shown in Figure 10. The reference value of the SOC was calculated according to the Ah integration method. It was worth noting that the Ah integration method showed a low estimation accuracy in practical application because it was greatly influenced by errors of initial value and capacity as well as the accumulative error of current measurement. By contrast, in a laboratory environment, the accuracy of the current measurement was high; experimental equipment was BTS-5V100A of Neware. The accuracy of the current measurement was within $\pm 0.1 \%$ [30], and Shen Ping's research on the current measurement error of equipment shows that when the relative error of the current measurement is $1 \%$, the theoretical error of the SOC error in the EKF is below $0.2 \%$ [31]; therefore, the accuracy of the current measurement can be ignored. The initial value and capacity of the SOC were 
known. Therefore, the part of the error can be ignored. In this case, the SOC calculated by applying the Ah integration method was accurate, which can be used as a reference value.

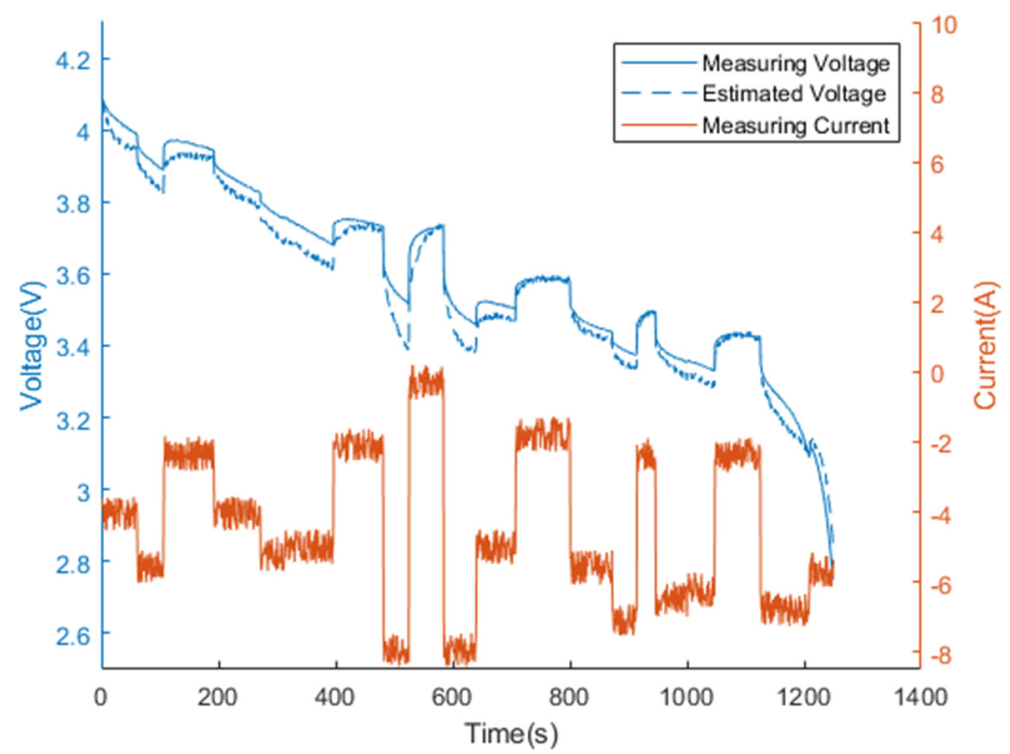

Figure 9. Measured voltages and currents on dynamic working conditions at $25^{\circ} \mathrm{C}$.

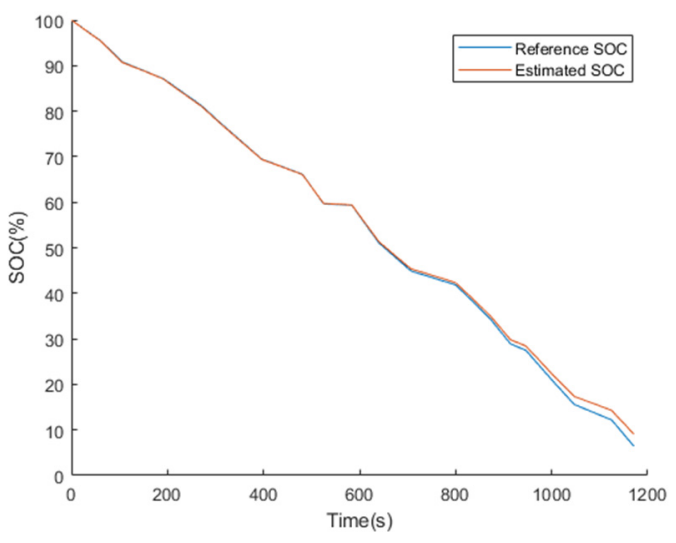

(a)

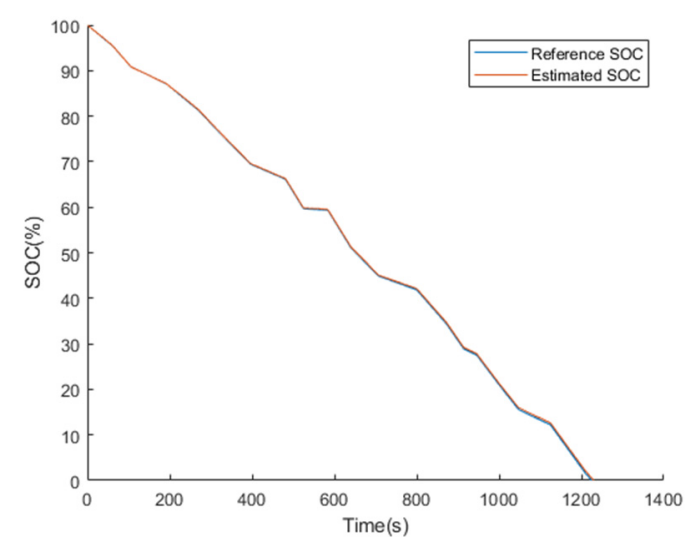

(c)

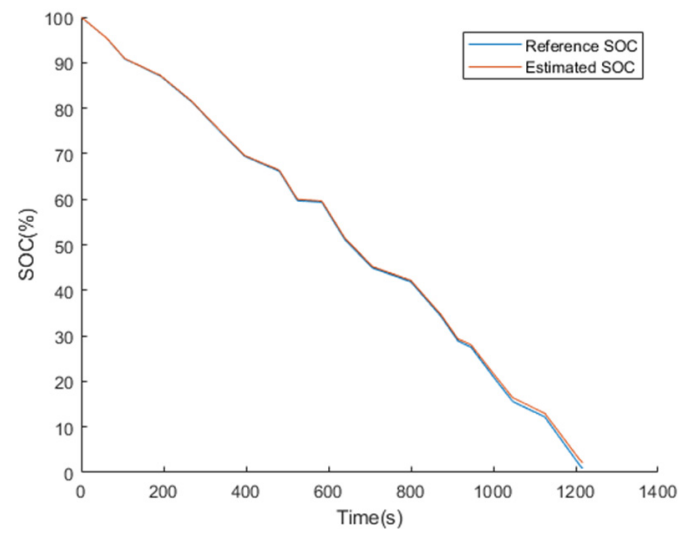

(b)

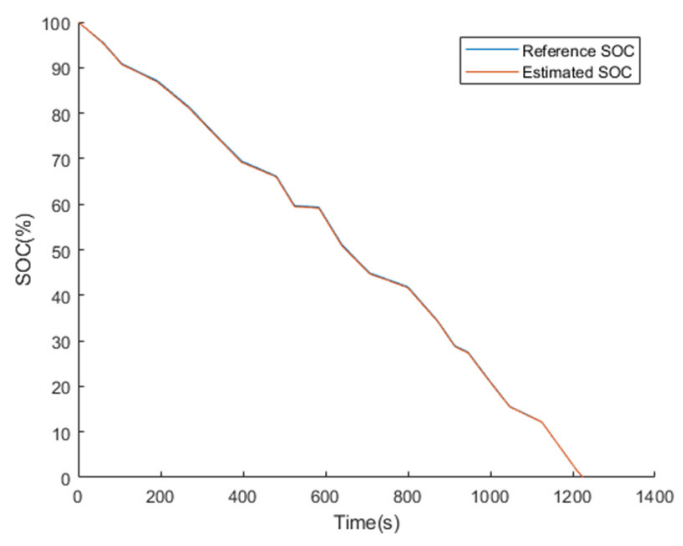

(d)

Figure 10. Estimated results of SOC: (a) $5{ }^{\circ} \mathrm{C}$; (b) $15^{\circ} \mathrm{C}$; (c) $25^{\circ} \mathrm{C}$; (d) $40{ }^{\circ} \mathrm{C}$. 
The SOC errors during the offline bench testing on practical working conditions are shown in Figure 11.

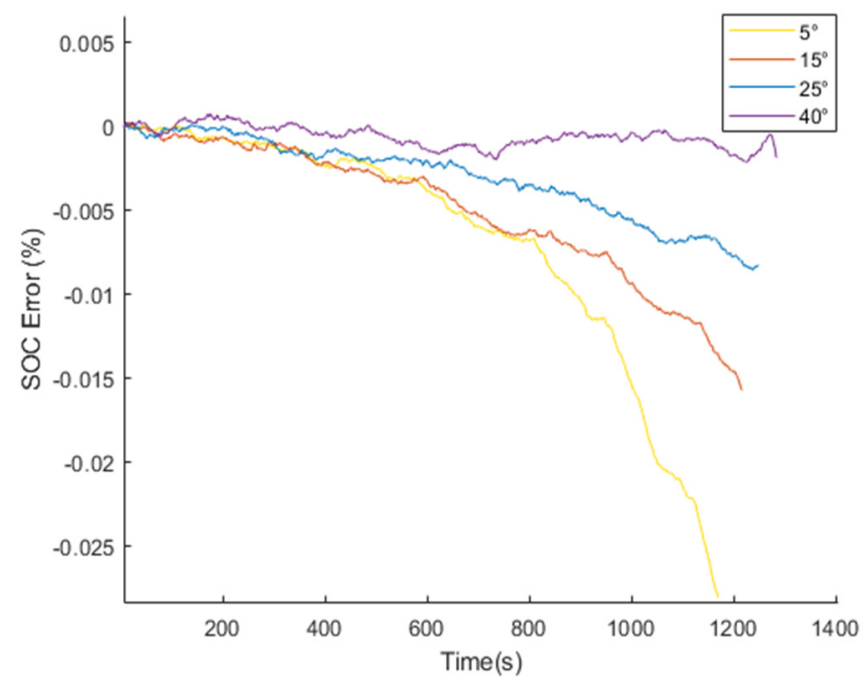

Figure 11. SOC estimation errors.

According to the verification results on practical and simulated working conditions, it can be seen that the maximum SOC estimation errors during high-temperature tests at $40{ }^{\circ} \mathrm{C}, 25^{\circ} \mathrm{C}, 15^{\circ} \mathrm{C}$, and $5{ }^{\circ} \mathrm{C}$ were $0.215 \%, 0.8627 \%, 1.5712 \%$, and $2.807 \%$, respectively.

Through analysis, it can be seen that at high ambient temperatures and room temperature, the capacities and model parameters obtained by using various estimation algorithms for the SOC were all accurate on the condition that batteries were not aged. Therefore, there was a high estimation accuracy of the SOC, with the error within $1 \%$. At a low temperature, the batteries were significantly polarized so that the accuracy of the battery model was low. Therefore, the estimation accuracy for SOC slightly decreased, with a maximum error of about $2.8 \%$.

\section{Conclusions}

At present, research on SOC estimation of power-type Li batteries, the power source of small-sized UAVs, is hardly reported. In the current study, the currently widely used methods for SOC estimation of Li batteries for vehicles were explored and compared to analyze their advantages and disadvantages. Afterward, by using the EKF algorithm, a closed-loop estimation method based on the equivalent circuit model, the SOC of power-type Li batteries was estimated.

By conducting offline bench testing at different temperatures and different working conditions, it was found that at high ambient pressures or room temperatures, the capacities and model parameters obtained by using estimation algorithms for the SOC based on the EKF were all accurate on the condition that batteries were not aged. Therefore, the estimation accuracy of the SOC was high, with the error below $1 \%$. At a low temperature, batteries were subjected to significant polarization, so that the battery model exhibited low accuracy. Thus, the estimation accuracy of the SOC slightly reduced, with the maximum error of about $2.8 \%$. EKF can still estimate the SOC of batteries relatively accurately. Therefore, EKF can be practically applied to estimate the SOC of power-type Li batteries of small-sized UAVs, thus improving the battery life of UAVs.

However, compared with offline bench experiments, the application of SOC estimation practically with EKF is difficult and it is not possible to guarantee high estimation accuracy due to battery aging, a wide range in temperature, initial value uncertainty, and inaccurate voltage and current measurement. Therefore, subsequent work should focus on analyzing the possible sources of error and the exact impacts on the SOC estimate to minimize errors. 
Author Contributions: H.Z. independently completed theoretical calculations, system design, and experimental verification. H.Z. carried out the research and analyzed the numerical data with guidance from M.Z. and X.L. H.Z. prepared the manuscript.

Funding: This research received no external funding.

Conflicts of Interest: The authors declare no conflict of interest.

\section{References}

1. Liang, B.; Wang, H.; Yuan, C. Design and Optimization of Power System for Small Electric UAV; School of Aeronautics, Northwestern Polytechnical University: Xian, China, 2010. (In Chinese)

2. Logan, M.; Chu, J.; Motter, M.; Carter, D.; Ol, M.; Zeune, C. Small UAV research and evolution in long endurance electric powered vehicles. In Proceedings of the AIAA Infotech@ Aerospace 2007 Conference and Exhibit, Rohnert Park, CA, USA, 7-10 May 2007.

3. Cavcar, M.; Cavcar, A. Optimum range and endurance of a piston propeller aircraft with cambered wing. J. Aircr. 2005, 42, 212-217. [CrossRef]

4. Wang, G.; Hu, Y.; Song, B.; Tan, C. Optimal design and endurance estimation of propulsion system for electricpowered unmanned vehicle. J. Aerosp. Power 2015, 30, 1834-1840, (In Chinese with English Abstract).

5. He, Y. Research on State Estimation Strategy and Management System Technology of Power Lithium Battery Pack; University of Science and Technology: Anhui, China, 2012. (In Chinese)

6. Schacht-Rodriguez, R.; Ortiz-Torres, G.; Garcia-Beltran, C.D.; Astorga-Zaragoza, C.M.; Ponsart, J.C.; Theilliol, D. SoC estimation using an Extended Kalman filter for UAV applications. In Proceedings of the 2017 International Conference on Unmanned Aircraft Systems (ICUAS), Miami, FL, USA, 13-16 June 2017; pp. 179-187.

7. Shen, P. Research on High-Precision Co-Estimation of Battery State based on Model; Tsinghua University: Beijing, China, 2018. (In Chinese)

8. Shen, P.; Ouyang, M.; Lu, L.; Li, J.; Feng, X. The co-estimation of state of charge, state of health, and state of function for lithium-ion batteries in electric vehicles. IEEE Trans. Veh. Technol. 2018, 67, 92-103. [CrossRef]

9. Lu, L.; Han, X.; Li, J.; Hua, J.; Ouyang, M. A review on the key issues for lithium-ion battery management in electric vehicles. J. Power Sources 2013, 226, 272-288. [CrossRef]

10. Guoliang, W.; Rengui, L.; Chunbo, Z.; Chan, C.C. State of charge estimation for NiMH battery based on electromotive force method. In Proceedings of the IEEE Vehicle Power and Propulsion Conference, Harbin, China, 3-5 September 2008; pp. 1-5.

11. Coleman, M.; Lee, C.K.; Zhu, C.; Hurley, W. State-of-Charge Determination from EMF Voltage Estimation: Using Impedance, Terminal Voltage, and Current for Lead-Acid and Lithium-Ion Batteries. IEEE Trans. Ind. Electron. 2007, 54, 2550-2557. [CrossRef]

12. Dang, X.; Yan, L.; Xu, K.; Wu, X.; Jiang, H.; Sun, H. Open-Circuit Voltage-Based State of Charge Estimation of Lithium-ion Battery Using Dual Neural Network Fusion Battery Model. Electrochim. Acta 2016, 188, 356-366. [CrossRef]

13. Hu, J.; Hu, J.; Lin, H.; Li, X.; Jiang, C.; Qiu, X.; Li, W. State-of-charge estimation for battery management system using optimized support vector machine for regression. J. Power Sources 2014, 269, 682-693. [CrossRef]

14. Han, X. Research on Mechanism Model and State Estimation of Lithium Ion Battery for Vehicles; Tsinghua University: Beijing, China, 2015. (In Chinese)

15. Hu, X.; Li, S.; Peng, H. A comparative study of equivalent circuit models for Li-ion batteries. J. Power Sources 2012, 198, 359-367. [CrossRef]

16. Nejad, S.; Gladwin, D.T.; Stone, D.A. A systematic review of lumped-parameter equivalent circuit models for real-time estimation of lithium-ion battery states. J. Power Sources 2016, 316, 183-196. [CrossRef]

17. Cheng, K.W.E.; Divakar, B.P.; Wu, H.; Ding, K.; Ho, H.F. Battery-Management System (BMS) and SOC Development for Electrical Vehicles. IEEE Trans. Veh. Technol. 2011, 60, 76-88. [CrossRef]

18. Plett, G.L. Extended Kalman filtering for battery management systems of LiPB-based HEV battery packs. J. Power Sources 2004, 134, 252-261. [CrossRef]

19. Plett, G.L. Extended Kalman filtering for battery management systems of LiPB-based HEV battery packs. J. Power Sources 2004, 134, 262-276. [CrossRef]

20. Hu, X.; Li, S.; Peng, H.; Sun, F. Robustness analysis of State-of-Charge estimation methods for two types of Li-ion batteries. J. Power Sources 2012, 217, 209-219. [CrossRef] 
21. Plett, G.L. Sigma-point Kalman filtering for battery management systems of LiPB-based HEV battery packs. J. Power Sources 2006, 161, 1356-1368. [CrossRef]

22. He, H.; Xiong, R.; Peng, J. Real-time estimation of battery state-of-charge with unscented Kalman filter and RTOS $\mu$ COS-II platform. Appl. Energy 2016, 162, 1410-1418. [CrossRef]

23. Li, J.; Klee Barillas, J.; Guenther, C.; Danzer, M.A. A comparative study of state of charge estimation algorithms for LiFePO4 batteries used in electric vehicles. J. Power Sources 2013, 230, 244-250. [CrossRef]

24. Hu, X.; Sun, F.; Zou, Y. Estimation of State of Charge of a Lithium-Ion Battery Pack for Electric Vehicles Using an Adaptive Luenberger Observer. Energies 2010, 3, 1586-1603. [CrossRef]

25. Zeng, Z.; Tian, J.; Li, D.; Tian, Y. An Online State of Charge Estimation Algorithm for Lithium-Ion Batteries Using an Improved Adaptive Cubature Kalman Filter. Energies 2018, 11, 59. [CrossRef]

26. Xiong, R.; Sun, F.; Chen, Z.; He, H. A data-driven multi-scale extended Kalman filtering based parameter and state estimation approach of lithium-ion polymer battery in electric vehicles. Appl. Energy 2014, 113, 463-476. [CrossRef]

27. Xia, B.; Lao, Z.; Zhang, R.; Tian, Y.; Chen, G.; Sun, Z.; Wang, W.; Sun, W.; Lai, Y.; Wang, M.; et al. Online Parameter Identification and State of Charge Estimation of Lithium-Ion Batteries Based on Forgetting Factor Recursive Least Squares and Nonlinear Kalman Filter. Energies 2018, 11, 3. [CrossRef]

28. IdAho National Laboratory. Battery Test Manual for Plug-In Hybrid Electric Vehicles; Idaho National Laboratory: Idaho Falls, ID, USA, 2010.

29. Sierra, G.; Orchard, M.; Goebel, K.; Kulkarni, C. Battery health management for small-size rotary-wing electric unmanned aerial vehicles: An efficient approach for constrained computing platforms. Reliab. Eng. Syst. Saf. 2019, 182, 166-178. [CrossRef]

30. Technical Specification Document of BTS-5V100A; Neware Electronics Co., Ltd.: Futian District, Shenzhen, China, 2019.

31. Shen, P.; Ouyang, M.; Han, X.; Feng, X.; Lu, L.; Li, J. Error Analysis of the Model-Based State-of-Charge Observer for Lithium-Ion Batteries. IEEE Trans. Veh. Technol. 2018, 67, 8055-8064. [CrossRef] 